\section{Lenalidomide before and after autologous stem cell transplantation for transplant-eligible patients of all ages in the randomized, phase III, Myeloma XI trial}

Graham H. Jackson, ${ }^{1}$ Faith E. Davies, ${ }^{2}$ Charlotte Pawlyn, ${ }^{3}$ David A. Cairns, ${ }^{4}$ Alina Striha, ${ }^{4}$ Corinne Collett, ${ }^{4}$ Anna Waterhouse, ${ }^{4}$ John R. Jones, ${ }^{5}$ Bhuvan Kishore, ${ }^{6}$ Mamta Garg, ${ }^{7}$ Cathy D. Williams, ${ }^{8}$ Kamaraj Karunanithi, ${ }^{9}$ Jindriska Lindsay, ${ }^{10}$ David Allotey, ${ }^{11}$ Salim Shafeek, ${ }^{12}$ Matthew W. Jenner, ${ }^{13}$ Gordon Cook, ${ }^{14}$ Nigel H. Russell, ${ }^{8}$ Martin F. Kaiser, ${ }^{3}$ Mark T. Drayson, ${ }^{15}$ Roger G. Owen, ${ }^{16}$ Walter M. Gregory ${ }^{4}$ and Gareth J. Morgan ${ }^{2}$ for the UK NCRI Haematological Oncology Clinical Studies Group

${ }^{1}$ Northern Institute for Cancer Research, Newcastle University, Newcastle upon Tyne, UK; ${ }^{2}$ Perlmutter Cancer Center, NYU Langone Health, New York, NY, USA; ${ }^{3}$ The Institute of Cancer Research and The Royal Marsden Hospital NHS Foundation Trust, London, UK; ${ }^{4}$ Clinical Trials Research Unit, Leeds Institute of Clinical Trials Research, University of Leeds, Leeds, UK; ${ }^{5}$ King's College Hospital NHS Foundation Trust, London, UK; ${ }^{6}$ Heart of England NHS Foundation Trust, Birmingham, UK; ' ${ }^{2}$ eicester Royal Infirmary, Leicester, UK; ${ }^{8}$ Centre for Clinical Haematology, Nottingham University Hospital, Nottingham, UK; 'University Hospital of North Midlands, Stoke-on-Trent, UK; ${ }^{10}$ East Kent Hospitals University NHS Foundation Trust, Canterbury, UK; ${ }^{11}$ Derby Teaching Hospitals NHS Foundation Trust, Derby, UK; ${ }^{12}$ Worcestershire Acute Hospitals NHS Trust, Worcester, UK; ${ }^{13}$ University Hospital Southampton NHS Foundation Trust, Southampton, UK; ${ }^{14}$ Section of Experimental Haematology, Leeds Institute of Cancer and Pathology, University of Leeds, Leeds, UK; ${ }^{15}$ Clinical Immunology, School of Immunity and Infection, University of Birmingham, Birmingham, UK and ${ }^{16} \mathrm{St}$ James's University Hospital, Haematological Malignancy Diagnostic Service (HMDS), Leeds, UK

\section{ABSTRACT}

1 he optimal way to use immunomodulatory drugs as components of induction and maintenance therapy for multiple myeloma is unresolved. We addressed this question in a large phase III randomized trial, Myeloma XI. Patients with newly diagnosed multiple myeloma $(n=2,042)$ were randomized to induction therapy with cyclophosphamide, thalidomide, and dexamethasone (CTD) or cyclophosphamide, lenalidomide, and dexamethasone (CRD). Additional intensification therapy with cyclophosphamide, bortezomib, and dexamethasone (CVD) was administered before autologous stem-cell transplantation to patients with a suboptimal response to induction therapy using a response-adapted approach. After receiving high-dose melphalan with autologous stem cell transplantation, eligible patients were further randomized to receive either lenalidomide alone or observation alone. Co-primary endpoints were progression-free survival (PFS) and overall survival (OS). The CRD regimen was associated with significantly longer PFS (median: 36 vs. 33 months; hazard ratio [HR], 0.85; 95\% confidence interval [CI]: 0.75-0.96; $P=0.0116$ ) and OS (3-year OS: $82.9 \%$ vs. $77.0 \%$; HR, 0.77; 95\% CI: $0.63-0.93 ; P=0.0072)$ compared with CTD. The PFS and OS results favored CRD over CTD across all subgroups, including patients with International Staging System stage III disease (HR for PFS, 0.73; 95\% CI: 0.58-0.93; HR for OS, 0.78; 95\% CI: 0.56-1.09), high-risk cytogenetics (HR for PFS, 0.60; 95\% CI: 0.43-0.84; HR for OS, 0.70; 95\% CI: $0.42-1.15$ ) and ultra-high-risk cytogenetics (HR for PFS, 0.67; 95\% CI: 0.411.11; HR for OS, 0.65 ; 95\% CI: 0.34-1.25). Among patients randomized to lenalidomide maintenance $(n=451)$ or observation $(n=377)$, maintenance therapy improved PFS (median: 50 vs. 28 months; HR, 0.47; 95\% CI: 0.37$0.60 ; P<0.0001)$. Optimal results for PFS and OS were achieved in the patients who received CRD induction and lenalidomide maintenance. The trial was registered with the EU Clinical Trials Register (EudraCT 2009010956-93) and ISRCTN49407852.
Ferrata Storti Foundation

Haematologica 2021

Volume 106(7):1957-1967

\section{Correspondence:}

GRAHAM H. JACKSON

graham.jackson@newcastle.ac.uk

Received: January 20, 2020.

Accepted: May 28, 2020.

Pre-published: June 4, 2020.

https://doi.org/10.3324/haematol.2020.247130

\section{(C)2021 Ferrata Storti Foundation}

Material published in Haematologica is covered by copyright. All rights are reserved to the Ferrata Storti Foundation. Use of published material is allowed under the following terms and conditions:

https://creativecommons.org/licenses/by-nc/4.0/legalcode. Copies of published material are allowed for personal or internal use. Sharing published material for non-commercial purposes is subject to the following conditions:

https://creativecommons.org/licenses/by-nc/4.0/legalcode, sect. 3. Reproducing and sharing published material for commercial purposes is not allowed without permission in writing from the publisher. 


\section{Introduction}

The introduction of novel agents, such as immunomodulatory drugs and proteasome inhibitors, has contributed to the recent dramatic improvements in outcomes observed for patients with multiple myeloma. ${ }^{1-3}$ Following induction, high-dose melphalan-based chemotherapy with autologous stem cell transplantation (ASCT) remains the standard of care for eligible patients. ${ }^{49}$ The optimal approach to induction therapy prior to ASCT and consolidation or maintenance after ASCT in this new era has not yet been defined. However, several principles have been established, including the value of using at least triplet combinations of agents that can induce deeper, longer remissions by targeting different clonal populations. ${ }^{10,11}$

The efficacy of immunomodulatory drugs in multiple myeloma has been linked to their mode of action. These drugs target the cereblon ubiquitin ligase complex, which leads to both tumoricidal effects early on and immunomodulatory effects beneficial for long-term tumor control. ${ }^{12-15}$ The immunomodulatory drugs thalidomide and lenalidomide are recognized as effective treatment options in both the induction ${ }^{7,9,10,16-18}$ and maintenance settings. ${ }^{6,19-21}$ Lenalidomide has fewer side effects than thalidomide, enabling long-term treatment and disease control. ${ }^{19-21}$ We have addressed how to optimize the use of these agents between induction and maintenance for patients receiving ASCT in a large, randomized trial (UK National Cancer Research Institute [NCRI] Myeloma XI).

\section{Methods}

The Myeloma XI study had a multifactorial design enabling the investigation of a number of pertinent clinical questions with adequate statistical control and power. Importantly, the influence of one phase of treatment or question on another could be separated and controlled for. This was achieved by stratifying the consolidation and maintenance randomizations for earlier treatment allocations. This report concentrates on induction and its interaction with maintenance therapy in the transplant-eligible population of patients within the trial. The other questions posed by the study are addressed in separate manuscripts.

\section{Study design and eligibility criteria}

The Myeloma XI trial was a phase III, open-label, parallelgroup, multi-arm, adaptive design trial with three randomization stages conducted at 110 National Health Service hospitals in England, Wales, and Scotland (see Online Supplementary Data for a list of study sites with principal investigators and number of patients recruited). Eligible patients were aged $\geq 18$ years and newly diagnosed with multiple myeloma. Exclusion criteria included previous or concurrent malignancies (including myelodysplastic syndromes), grade $\geq 2$ peripheral neuropathy, acute renal failure (unresponsive to up to $72 \mathrm{~h}$ of rehydration, characterized by creatinine $>500 \mu \mathrm{mol} / \mathrm{L}$ or urine output $<400 \mathrm{~mL} /$ day or requiring dialysis), and active or prior hepatitis $\mathrm{C}$ infection.

The trial design included an intensive treatment pathway for transplant-eligible patients and a less intensive treatment pathway for transplant-ineligible patients. Strict age limits were deliberately avoided so that fit, older patients could receive intensive therapy and ASCT. The decision of which treatment pathway to undertake was made on an individual patient basis taking into account performance status, clinician judgment, and patient preference.

Transplant-eligible patients were randomized on a 1:1 basis to cyclophosphamide, lenalidomide, and dexamethasone (CRD) or cyclophosphamide, thalidomide, and dexamethasone (CTD) (induction randomization), stratified according to certain factors (Online Supplementary Methods). Patients received a minimum of four cycles in the absence of progressive disease, and treatment continued until maximum response was achieved.

Additional intensification therapy before ASCT was administered to patients with a suboptimal response to induction therapy using a response-adapted approach: patients with stable disease after induction therapy or those with progressive disease at any time during induction therapy received a maximum of eight cycles of cyclophosphamide, bortezomib, and dexamethasone (CVD); patients with a minimal or partial response were randomized (1:1) to CVD or no CVD. Patients with a very good partial response or complete response received no additional therapy before ASCT. The results of the intensification randomization have been published elsewhere. ${ }^{22}$

Three months after ASCT, eligible patients were randomized to observation or to maintenance therapy with lenalidomide alone, or in combination with vorinostat until unacceptable toxicity or progressive disease. Patients were excluded from maintenance randomization if they did not respond to CRD induction, had no response to any prior study treatment, had progressive disease, or relapsed after achieving a complete response. Randomized patients were stratified according to treatment center and previous randomization group(s). The results of the maintenance randomization have been published elsewhere. ${ }^{23}$

Further details on the dose and schedule of all study treatments are provided in Online Supplementary Table S1, and a flow diagram of the CRD and CTD groups of patients is shown in Online Supplementary Figure S1.

The study was approved by the national ethics review board (National Research Ethics Service, London, UK), institutional review boards of the participating centers, and the competent regulatory authority (Medicines and Healthcare Products Regulatory Agency, London, UK). All patients provided written informed consent. The trial was registered with the EU Clinical Trials Register (EudraCT number, 2009-010956-93) and ISRCTN49407852.

\section{Study endpoints and statistical analysis}

The co-primary endpoints were progression-free survival (PFS) and overall survival (OS). Secondary endpoints included PFS-Two (PFS2), response, and safety. Further details regarding the statistical analysis are provided in the Online Supplementary Material.

\section{Results}

\section{Patients}

Between May 26, 2010 and April 20, 2016, 2,042 transplant-eligible patients underwent induction randomization (Online Supplementary Figure S1). Baseline characteristics were well balanced between the two treatment groups (Table 1). The median age of all the patients was 61 years (range, $28-75$ years), $60 \%$ of the patients were male, and $24 \%$ had International Staging System (ISS) stage III disease. Of the $836(40.9 \%)$ patients for whom genetic risk could be calculated, 266 (31.8\%) had high-risk and $111(13.3 \%)$ had ultra-high-risk cytogenetics. The median duration of follow-up since study entry was 36.3 months (interquartile range [IOR], 23.0-48.5 months).

\section{Induction randomization results \\ Progression-free survival and overall survival}

Disease progression or death occurred in 456 patients in 
Table 1. Patients' characteristics according to induction regimen.

\begin{tabular}{|c|c|c|}
\hline Characteristic & CRD $(n=1,021)$ & CTD $(n=1,021)$ \\
\hline Median age (range), years & $61(28-75)$ & $61(29-74)$ \\
\hline \multicolumn{3}{|l|}{ Age group, n (\%) } \\
\hline$\leq 65$ years & $772(75.6)$ & $754(73.8)$ \\
\hline$>65$ years & $249(24.4)$ & $267(26.2)$ \\
\hline \multicolumn{3}{|l|}{ Sex, n (\%) } \\
\hline Male & $610(59.7)$ & $611(59.8)$ \\
\hline Female & $411(40.3)$ & $410(40.2)$ \\
\hline \multicolumn{3}{|l|}{ Ethnicity, n (\%) } \\
\hline White & $938(91.9)$ & $937(91.8)$ \\
\hline Black (Black Caribbean, Black African, other) & $21(2.1)$ & $14(1.4)$ \\
\hline Asian (Indian, Pakistani, Bangladeshi, other) & $28(2.7)$ & $27(2.6)$ \\
\hline Other & $10(0.9)$ & $14(1.4)$ \\
\hline Unknown & $24(2.4)$ & $29(2.8)$ \\
\hline \multicolumn{3}{|l|}{ WHO performance status, n (\%) } \\
\hline 0 & $421(41.2)$ & $439(43.0)$ \\
\hline 1 & $363(35.6)$ & $367(35.9)$ \\
\hline 2 & $119(11.7)$ & $135(13.2)$ \\
\hline$\geq 3$ & $53(5.2)$ & $34(3.3)$ \\
\hline Unknown & $65(6.4)$ & $46(4.5)$ \\
\hline \multicolumn{3}{|l|}{ Immunoglobin subtype, $\mathrm{n}(\%)$} \\
\hline $\operatorname{IgG}$ & $633(62.0)$ & $600(58.8)$ \\
\hline IgA & $220(21.5)$ & $269(26.3)$ \\
\hline IgM & $4(0.4)$ & $4(0.4)$ \\
\hline $\operatorname{IgD}$ & $12(1.2)$ & $9(0.9)$ \\
\hline Light chain only & $139(13.6)$ & $127(12.4)$ \\
\hline Non-secretor & $6(0.6)$ & $7(0.7)$ \\
\hline Unknown & $7(0.7)$ & $5(0.5)$ \\
\hline \multicolumn{3}{|l|}{ ISS stage, $\mathrm{n}(\%)$} \\
\hline I & $301(29.5)$ & $306(30.0)$ \\
\hline II & $392(38.4)$ & $388(38.0)$ \\
\hline III & $246(24.1)$ & $253(24.8)$ \\
\hline Unknown & $82(8.0)$ & $74(7.2)$ \\
\hline Median serum creatinine (range), $\mathrm{mol} / \mathrm{L}$ & $85.0(28.0-825.0)$ & $83.0(30.0-897.0)$ \\
\hline Unknown, n (\%) & $9(8.8)$ & $7(6.9)$ \\
\hline Median lactate dehydrogenase (range), IU/L & $262.0(3.0-2519.0)$ & $273.0(0.0-3550.0)$ \\
\hline Unknown, n (\%) & $228(22.3)$ & $215(21.1)$ \\
\hline \multicolumn{3}{|l|}{ CVD randomization after MR/PR, n (\%) } \\
\hline Allocated to CVD & $85(8.3)$ & $98(9.6)$ \\
\hline Allocated to no CVD & $82(8.0)$ & $102(10.0)$ \\
\hline Received CVD after SD/PD, n (\%) & $35(3.4)$ & $38(3.7)$ \\
\hline \multicolumn{3}{|l|}{ Maintenance treatment, n (\%) } \\
\hline Lenalidomide & $230(22.5)$ & $221(21.6)$ \\
\hline Lenalidomide plus vorinostat & $103(10.1)$ & $93(9.1)$ \\
\hline Observation & $190(18.6)$ & $187(18.3)$ \\
\hline Cytogenetic data available, $\mathrm{n}(\%)$ & $414(40.5)$ & $422(41.3)$ \\
\hline \multicolumn{3}{|c|}{ Cytogenetic lesions, $\mathrm{n}$ (\% of those with data available) } \\
\hline $\mathrm{t}(4 ; 14)$ & $56(13.5)$ & $70(16.6)$ \\
\hline $\mathrm{t}(14 ; 16)$ & $8(1.9)$ & $12(2.8)$ \\
\hline $\mathrm{t}(14 ; 20)$ & $3(0.7)$ & $2(0.5)$ \\
\hline $\operatorname{del}(17 p)$ & $31(7.5)$ & $42(10.0)$ \\
\hline gain(lq) & $137(33.1)$ & $136(32.2)$ \\
\hline \multicolumn{3}{|c|}{ Cytogenetic risk category, $\mathrm{n}$ (\% of those with data available) } \\
\hline Standard & $223(53.9)$ & $236(55.9)$ \\
\hline High* & $149(36.0)$ & $117(27.7)$ \\
\hline Ultra-high $^{\dagger}$ & $42(10.1)$ & $69(16.4)$ \\
\hline
\end{tabular}

CRD: cyclophosphamide, lenalidomide, and dexamethasone; CTD: cyclophosphamide, thalidomide, and dexamethasone; CVD: cyclophosphamide, bortezomib, and dexamethasone; Ig: immunoglobulin; ISS: International Staging System; MR: minimal response; PD: progressive disease; PR: partial response; SD: stable disease; WHO: World Health Organization. *High risk defined as the presence of any one of $\mathrm{t}(4 ; 14), \mathrm{t}(14 ; 16), \mathrm{t}(14 ; 20)$, del(17p), or gain(1q). ${ }^{\dagger}$ Ultra-high risk defined as the presence of more than one lesion. 
the CRD group and in 509 patients in the CTD group. The $\mathrm{CRD}$ regimen was associated with significantly longer PFS than the CTD regimen (hazard ratio [HR], 0.85; 95\% confidence interval [CI]: 0.75-0.96; $P=0.0116$ ) (Figure 1A). The median PFS was 36 months (95\% Cl: 33-39) with CRD and 33 months (95\% CI: 31-35) with CTD. The median overall survival has not yet been reached with the current follow-up. Death occurred in 185 patients in the CRD group and in 230 patients in the CTD group. There was a statistically significant difference in OS favoring CRD (HR, 0.77; 95\% CI: 0.63-0.93; $P=0.0072$ ) (Figure 1B). The 3-year OS rate was $82.9 \%$ (95\% Cl: 80.2-85.7) with CRD and $77.0 \%$ (95\% CI: 73.9-80.0) with CTD.
Subgroup analyses indicated that PFS and OS were better with CRD than with CTD across all subgroups (Figure 2). In the subset of patients with ISS stage III disease, CRD was superior to CTD for PFS (HR, 0.73; 95\% CI: 0.58$0.93)$ and there was a trend toward improved OS (HR, 0.78; 95\% CI: 0.56-1.09). In each case, there was no evidence of heterogeneity of treatment effect (PFS: $P=0.2645$; OS: $P=0.7606$ ) (Figure 2). Similar results were seen in the subgroup of patients with high-risk cytogenetics (HR for PFS, 0.60; 95\% CI: 0.43-0.84; HR for OS, 0.70; 95\% CI: $0.42-1.15$ ) and ultra-high risk cytogenetics (HR for PFS, 0.67; 95\% CI: 0.41-1.11; $P=0.6164$; HR for OS, $0.65 ; 95 \%$ CI: $0.34-1.25 ; P=0.8131)$, with no significant heterogeneity
A

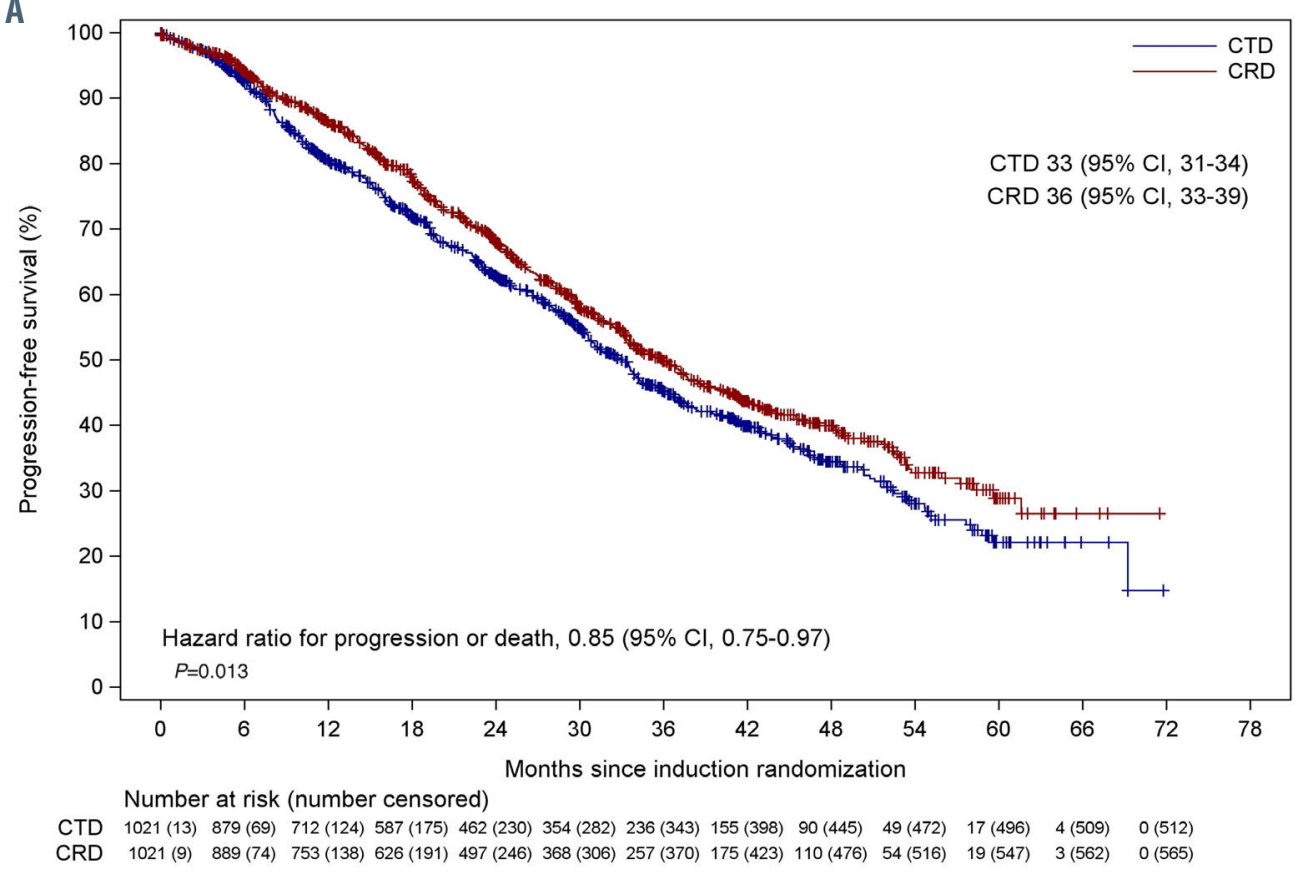

B

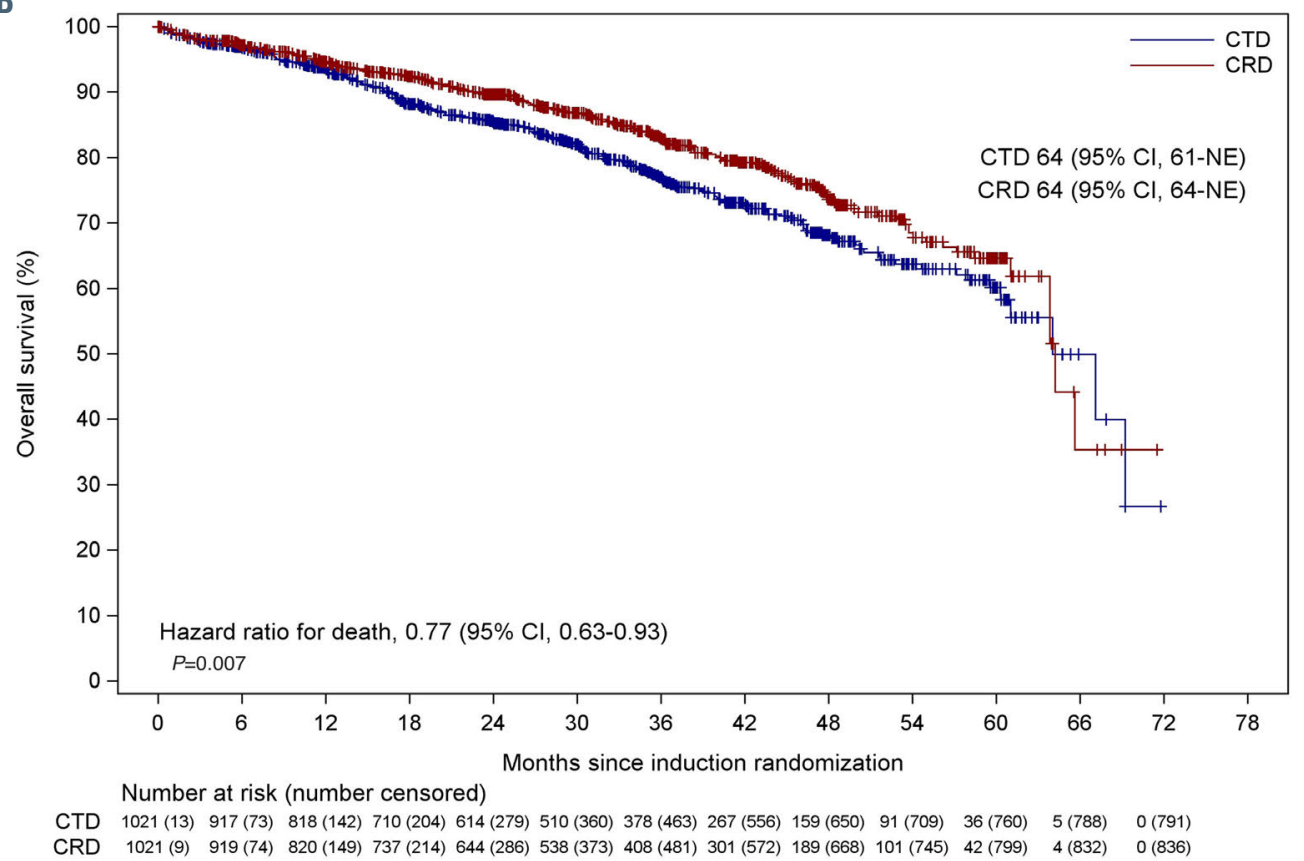

Figure 1. Outcomes according to induction regimen. (A) Progression-free survival and (B) overall survival, with dashed line showing the median. CRD: cyclophosphamide, lenalidomide, and dexamethasone; CTD: cyclophosphamide, thalidomide, and dexamethasone; $95 \% \mathrm{Cl}$ : $95 \%$ confidence interval. 
of treatment effect observed (Figure 2).

PFS2, a secondary endpoint, was also analyzed. CRD was associated with significantly longer PFS2 than was CTD (HR, 0.76; 95\% CI: 0.65-0.90; P=0.001) (Online Supplementary Figure S2). The median PFS2 was 59 months (95\% Cl: 55-63) with CRD and 54 months (95\% CI: 4960) with CTD.

\section{Response}

After induction triplet therapy, the proportion of patients with a very good partial response or better was significantly higher with CRD than with CTD $(60.4 \%$ vs. $52.9 \% ; P=0.0006$ ) (Table 2). The odds ratio (OR) of 1.37 (95\% CI: $1.15-1.65)$ indicates a $37 \%$ increase in the odds of achieving a deep remission in the CRD group than in the CTD group. After ASCT, the proportion of patients achieving a very good partial response or better remained higher in the CRD group than in the CTD group, but the difference was not statistically significant $(81.5 \%$ vs. 76.9\%; OR, 1.25; 95\% CI: 0.94-1.66; $P=0.1277$ ) (Table 2).

Due to the lower induction response rate with CTD compared with CRD, more patients underwent CVD intensification as per protocol (CRD, $11.8 \%$ vs. CTD, $13.3 \%)$. The interaction between induction therapy and CVD was therefore examined further. Counterfactual estimates of the survivor function if CVD rescue treatment was not received by any patients maintained differences in median PFS (CRD: 36 months [95\% CI: 33-39] vs. CTD: 33 months [95\% CI: 30-34]) (Online Supplementary Figure S3A) and 3-year OS rate (CRD: 82.9\% [95\% CI: 80.0-85.5] vs. CTD: $76.3 \% \quad[95 \%$ CI: 73.0-79.2]) (Online Supplementary Figure S3B). Similar counterfactual estimates obtained in the scenario in which patients randomized to no CVD after partial/minimal response were treated with CVD provided similar estimates for median PFS (CRD: 36 months [95\% CI: 33-39] vs. CTD: 33 months [95\% CI: 3136) (Online Supplementary Figure S3C) and 3-year OS rate (CRD: 83.1\% [95\% CI: 80.2-85.6] vs. CTD: 77.3\% [95\% CI: 74.1-80.2]) (Online Supplementary Figure S3D). After adjustment for the effect of CVD treatment in a counterfactual analysis, the hazard ratios for PFS and OS were 0.82 (95\% CI: $0.69-0.96)$ and 0.69 (95\% CI: $0.53-0.91)$, respectively. This suggests a greater treatment effect of
CRD induction treatment on PFS and particularly OS than apparent with the unadjusted intention-to-treat analysis (Online Supplementary Figure $S 3 A$ and $S 3 B$, respectively). The full results of the CVD intensification randomization have been presented elsewhere. ${ }^{22}$

\section{Safety}

The median number of cycles of induction therapy delivered was five (range, 1-18) for CRD and five (range, 1-12) for CTD. The median percentage of minimum protocol-defined delivered dose of lenalidomide and thalidomide during induction therapy was $116.7 \%$ (IOR, 96.4150.0 ) and $100.0 \%$ (IOR, 71.4-128.6), respectively. Lenalidomide dose modifications occurred in 391 (38.3\%) patients who received CRD induction therapy, and thalidomide dose modifications occurred in $751(73.6 \%)$ patients who received CTD induction therapy. The rate of discontinuation of induction therapy due to adverse events was similar with CRD and CTD (51 patients $[5.0 \%]$ and 68 patients $[6.7 \%]$, respectively). Overall, $64.4 \%$ of patients proceeded to ASCT following induction with or without intensification. There was no difference in the proportion of patients undergoing ASCT between those receiving CTD (63.3\%) or CRD (65.5\%) induction suggesting this was not due to induction-related toxicity. The most common reason for not proceeding was "Patient not fit/clinician's decision" in $36.1 \%$ of cases.

Differences in the safety profile of CRD and CTD were consistent with the known side effects of lenalidomide and thalidomide (Table 3). In general, CRD was associated with a higher rate of grade $\geq 3$ neutropenia $(22.3 \%$ vs. $11.7 \%$ ) and diarrhea $(2.6 \%$ vs. $1.0 \%)$, whereas CTD was associated with a higher rate of grade $\geq 3$ peripheral sensory neuropathy $(1.5 \%$ vs. $0.6 \%)$ and constipation $(1.9 \%$ vs. $0.8 \%$ ). The incidence of deep vein thrombosis was $5.7 \%$ in the CRD group and $4.8 \%$ in the CTD group; pulmonary embolism was reported in $3.2 \%$ and $4.9 \%$ of patients, respectively.

The 3-year cumulative incidence of invasive second primary malignancies (SPM) was low and comparable between CRD and CTD (2.9\% [95\% CI: 1.7-4.1] vs. $1.5 \%$ [95\% CI: $0.6-2.4] ; \quad H R, \quad 1.60$ [95\% CI: $0.87-2.93]$; $P=0.1311)$. The SPM incidence rate per 100 patient-years

Table 2. Response rates after induction and autologous stem-cell transplantation.

\begin{tabular}{|c|c|c|c|c|}
\hline & Response fo & tion therapy & Resp & ASCT \\
\hline Response, n (\%) & $\begin{array}{c}\text { CRD } \\
(n=1,021)\end{array}$ & $\begin{array}{c}\text { CTD } \\
(n=1,021)\end{array}$ & $\begin{array}{c}\text { CRD } \\
(n=628)\end{array}$ & $\begin{array}{c}\text { CTD } \\
(n=603)\end{array}$ \\
\hline CR or VGPR & $617(60.4)$ & $540(52.9)$ & $512(81.5)$ & $464(76.9)$ \\
\hline CR & $87(8.5)$ & $61(6.0)$ & 149 (23.7) & $122(20.2)$ \\
\hline CR w/o BM & 297 (29.1) & 223 (21.8) & 218 (34.7) & $214(35.5)$ \\
\hline VGPR & $233(22.8)$ & $256(25.1)$ & $145(23.1)$ & $128(21.2)$ \\
\hline PR or MR & $297(29.1)$ & $348(34.1)$ & $95(15.1)$ & $102(16.9)$ \\
\hline PR & $261(25.6)$ & $301(29.5)$ & $94(15.0)$ & $98(16.3)$ \\
\hline MR & $36(3.5)$ & $47(4.6)$ & $1(0.2)$ & $4(0.7)$ \\
\hline SD or PD & $32(3.1)$ & $43(4.2)$ & $11(1.8)$ & $10(1.7)$ \\
\hline SD & $8(0.8)$ & $8(0.8)$ & $0(0.0)$ & $0(0.0)$ \\
\hline PD & $24(2.4)$ & $35(3.4)$ & $11(1.8)$ & $10(1.7)$ \\
\hline Death within 100 days after ASCT & $13(1.3)$ & $17(1.7)$ & $1(0.2)$ & $6(1.0)$ \\
\hline Unknown & $57(5.6)$ & $61(6.0)$ & $9(0.9)$ & $21(2.1)$ \\
\hline
\end{tabular}

ASCT: autologous stem cell transplantation; CR: complete response; CR w/o BM: complete response by immunological criteria without confirmation by bone marrow; CRD: cyclophosphamide, lenalidomide, and dexamethasone; CTD: cyclophosphamide, thalidomide, and dexamethasone; MR: minimal response; PD: progressive disease; PR: partial response; SD: stable disease;VGPR: very good partial response. 
was 1.2 (95\% CI: 0.8-1.7) in the CRD group and $0.9(95 \%$ CI: $0.6-1.3)$ in the CTD group.

The incidence of serious adverse events during induction was similar with CRD and CTD $(59.0 \%$ vs. $57.7 \%)$. Infection accounted for nearly half of all serious adverse events reported during induction $(45.2 \%$ for CRD vs. $46.4 \%$ for CTD). Fatal adverse events occurred in six patients in the CRD group and in three patients in the CTD group. Of the nine patients with grade 5 adverse events, one had three concurrent events (renal failure, liver failure, and sepsis), one had two concurrent events (small bowel obstruction and sepsis), and the remaining seven patients had one event each (pneumonia [n=2]; sepsis $[\mathrm{n}=2]$; collapse/syncope $[\mathrm{n}=2]$; lower respiratory tract infection [ $\mathrm{n}=1]$; hepatitis encephalopathy $[\mathrm{n}=1]$ ).

\section{Interaction of lenalidomide induction and maintenance}

Following ASCT, patients were randomized between maintenance lenalidomide and observation, giving us the opportunity to explore the interaction between induction and maintenance agents in this setting. Of the 2,042 transplant-eligible patients who entered the first randomization, 1,024 entered the maintenance phase and were randomized to lenalidomide alone $(\mathrm{n}=451)$, to lenalidomide
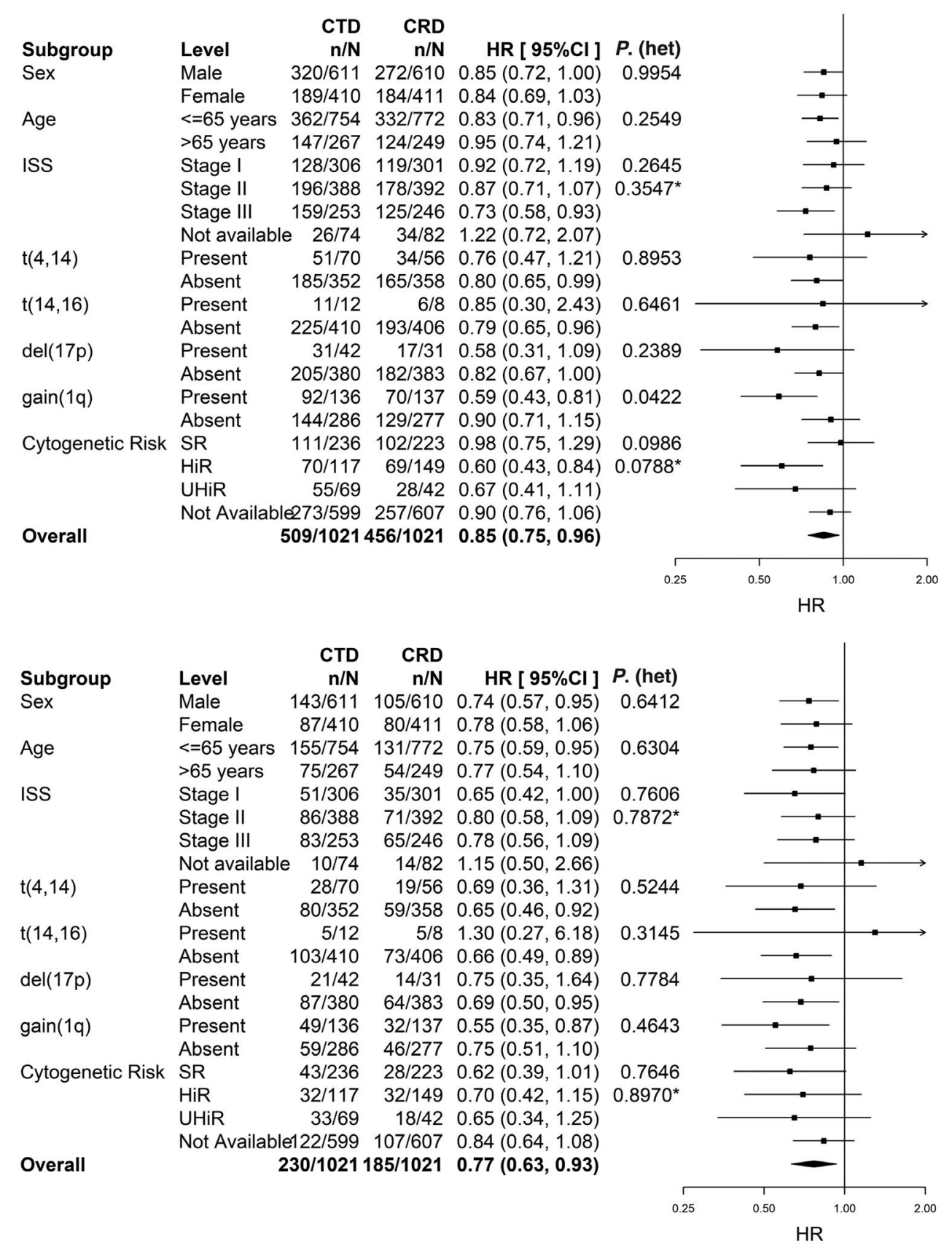

Figure 2. Outcomes according to induction regimen in selected subgroups. (A) Progression-free survival and (B) overall survival; Hazard ratio <1.00 favors CRD. * Likelihood ratio test for heterogeneity of effect among patients with subgroup data available. Cl: confidence interval; CRD: cyclophosphamide, lenalidomide, and dexamethasone; CTD: cyclophosphamide, thalidomide, and dexamethasone; het: heterogeneity; HiR: high risk; HR: hazard ratio; ISS: International Staging System; SR: standard risk; UHiR: ultra-high risk. 
plus vorinostat ( $\mathrm{n}=196$, not included in this further analysis), or to observation ( $n=377)$. The baseline characteristics of patients undergoing maintenance randomization were well balanced between the two treatment groups (Online Supplementary Table S2). Approximately half of patients in both the lenalidomide and observation groups had received CRD as induction therapy (230 of 451 [51.0\%] in the lenalidomide group; 190 of 377 [50.4\%] in the observation group). Lenalidomide maintenance was associated with significantly longer PFS and OS compared with observation in transplant-eligible patients (median: 50 vs. 28 months; HR, 0.47; 95\% CI: $0.37-0.60 ; P<0.0001$ at a median follow-up of 27.2 [IOR, 12.8-42.0] months).

In a post-hoc exploratory analysis, the longest PFS was

A

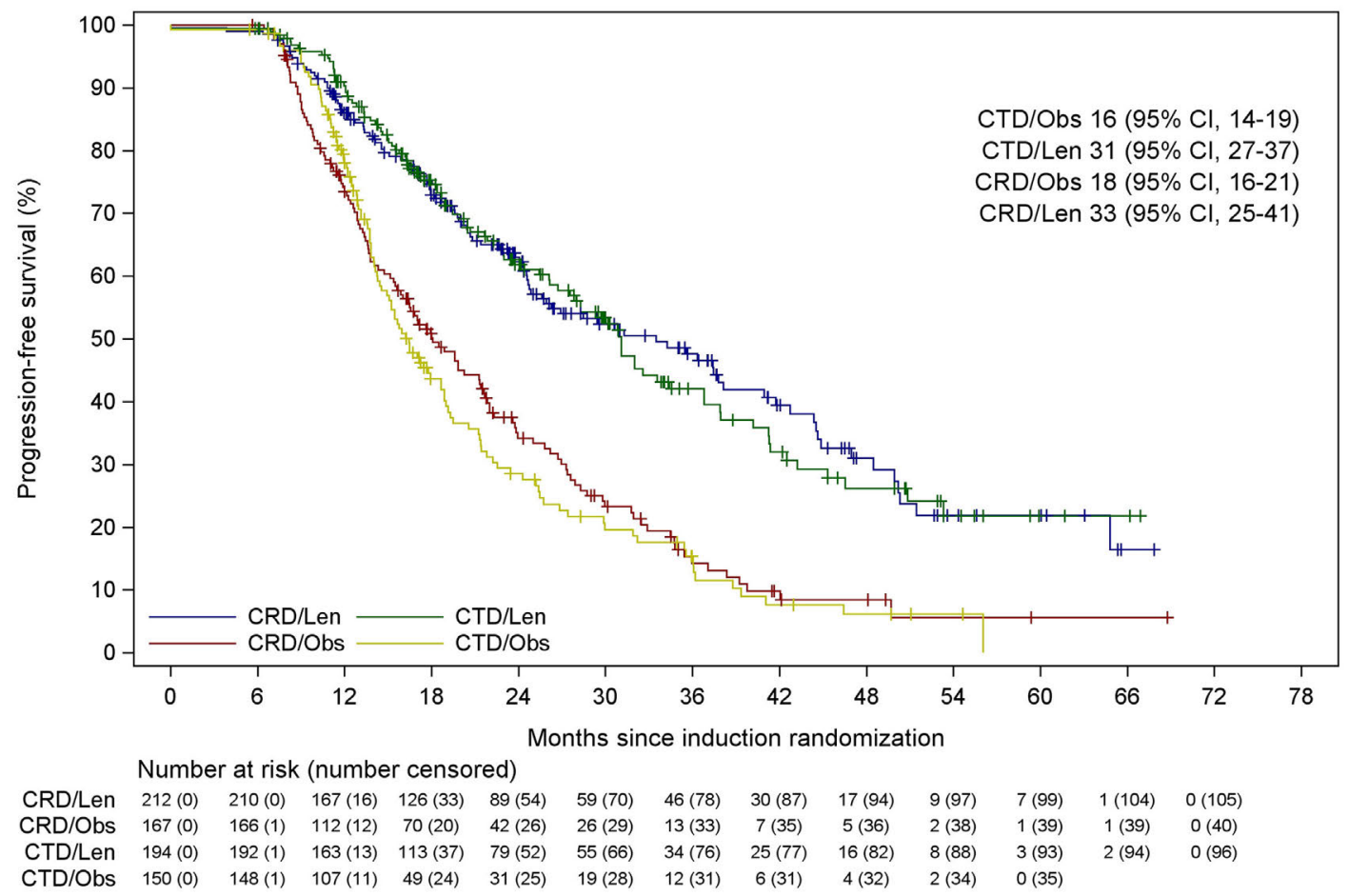

B

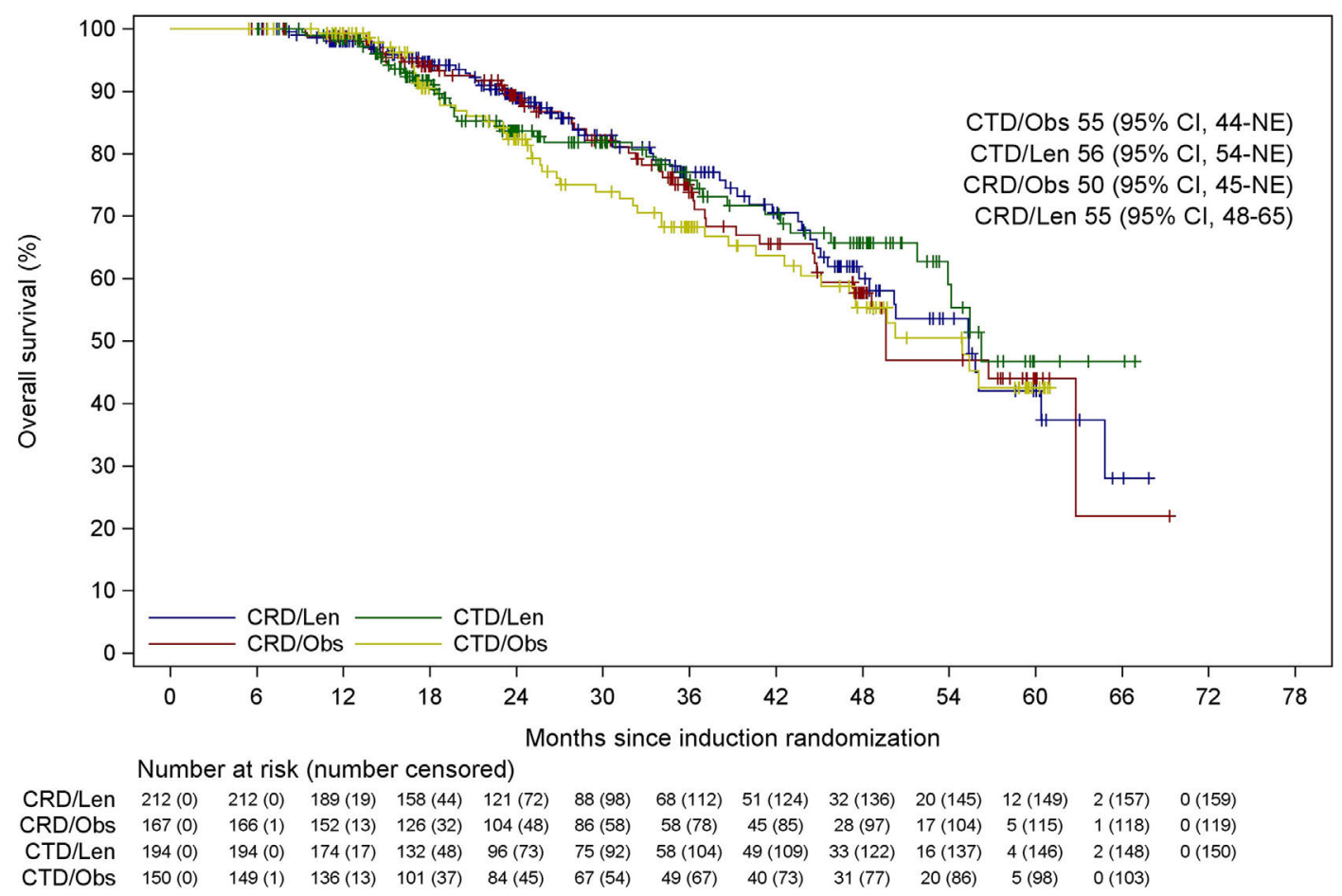

Figure 3. Outcomes according to induction and maintenance treatment. (A) Progression-free survival and (B) overall survival. CRD: cyclophosphamide, lenalidomide, and dexamethasone; CTD: cyclophosphamide, thalidomide, and dexamethasone; Obs: observation; Len: lenalidomide. 
observed in patients who received CRD induction and lenalidomide maintenance. The median PFS in this group was not reached, while it was 49 months in those who received CTD and lenalidomide maintenance, 32 months in those who received CTD and observation, and 24 months in those who received CRD and observation (Figure 3A). Similarly, the longest OS was observed in patients who received CRD induction and lenalidomide maintenance. The median OS was not reached in any group, but 3-year OS rates were $92.3 \%$ for those who received $\mathrm{CRD}$ induction with lenalidomide maintenance, $89.0 \%$ in those who received CTD and lenalidomide maintenance, $86.0 \%$ in those who received CTD and observation, and $90.3 \%$ in those who received CRD and observation (Figure 3B).

\section{Discussion}

This is the largest study to evaluate the CRD regimen as induction therapy before ASCT in patients with multiple myeloma. We show that it is associated with excellent efficacy and safety data and the results are consistent with prior studies evaluating either CTD,,$^{17,18,24} \mathrm{CRD}$ as induction therapy, ${ }^{25}$ or CRD as treatment in the relapsed/refractory disease setting. ${ }^{26}$

A direct comparison of thalidomide and lenalidomide as the immunomodulatory component of induction therapy has not been previously undertaken in the context of a randomized trial for transplant-eligible newly diagnosed myeloma patients. Our results demonstrate the superiority of lenalidomide over thalidomide both in terms of efficacy and tolerability in the context of combination with an alkylating agent (cyclophosphamide), supporting the findings of previous non-randomized analyses. ${ }^{27,28}$ Previous randomized studies in patients not eligible for stem-cell transplant have compared thalidomide to lenalidomide in combination with the alkylating agent melphalan..$^{29,30}$ In these studies no difference between lenalidomide and thalidomide in terms of response, progression-free or overall survival was identified. The differences between these prior studies and the finding from Myeloma XI might be explained by the different patient population or the different alkylating agent, cyclophosphamide, which may be better tolerated than melphalan.

Response rates obtained with CRD in the current study were good: $60 \%$ of patients achieved at least a very good partial response after induction and $82 \%$ did so after ASCT. This compares favorably with other novel-agent-based triplet induction therapies, including bortezomib, doxorubicin, and dexamethasone (VAD), ${ }^{31,82}$ $\mathrm{CVD},{ }^{32}$ bortezomib, thalidomide, and dexamethasone (VTD), ${ }^{5,10,33,34}$ and even the immunomodulatory drug/proteasome inhibitor regimen bortezomib, lenalidomide, and dexamethasone (VRD) $)^{9,35}$ (Online Supplementary Table S3). However there are many caveats when trying to compare results across trials. Particularly in comparing response rates it should be noted that patients in Myeloma XI received induction until maximum response rather than for a fixed duration and this may have led to deeper responses prior to transplantation than in other studies. Although immunomodulatory drug and proteasome inhibitor combinations (e.g. VTD/VRD) have recently become widely used in the European Union and
USA this was not the situation when the study was initially implemented. At that time either an immunomodulatory-based regimen or a proteasome inhibitor-based regimen (e.g., MPV or VD) was used. The standard of care in the UK, as in a number of other countries, was CTD. The addition of a proteasome inhibitor to induction regimens offers the potential to target immunomodulatory agent-resistant subclones of disease with a second novel agent. This concept was explored in the intensification randomization aspect of the study which has been previously reported ${ }^{22}$ and demonstrated that intensification treatment with CVD significantly improved PFS in patients with newly diagnosed multiple myeloma and a suboptimal response to immunomodulatory induction therapy compared with no intensification treatment.

The combination of a fourth agent with a different mechanism of action to induction, such as daratumumab plus VTD (Dara-VTD) investigated in the recently published Cassiopeia trial, is able to induce even deeper responses, with $83 \%$ of patients achieving at least very good partial response. ${ }^{36}$ PFS was longer in patients treated with Dara-VTD than in those treated with VTD alone, suggesting the addition of further agents to active triplets can improve outcomes yet further. In contrast, however, $\mathrm{CRD}$ offers an all oral regimen requiring only one hospital visit per month and including only one more expensive agent, lenalidomide. As such it is comparatively easier to deliver and likely to be cheaper in terms of both drug and administration costs. The lower incidence of peripheral neuropathy seen with CRD than that seen with combinations including bortezomib and/or thalidomide may also be beneficial for some patients.

The Myeloma XI data support the continued use of ASCT, since in a previous study of CRD without ASCT, the median PFS was 28.6 months, which is shorter than that achieved with CRD and ASCT in the Myeloma XI trial (36 months). Similarly, in the IFM 2009 study comparing VRD with or without ASCT, the combination of VRD and ASCT led to significantly better PFS than VRD alone (median: 50 vs. 36 months; $P<0.001$ ). ${ }^{9}$ The median OS in that study was similar in both groups, likely due to the fact that $79 \%$ of patients assigned to VRD alone received salvage ASCT at relapse and the short current follow-up. These findings and data from several other studies suggest a complementary role for novel agents and ASCT.

We have shown that treatment with lenalidomide maintenance therapy after ASCT is associated with improved PFS and OS, a finding consistent with other reports. ${ }^{6,19,20,37}$ We show that in Myeloma XI, the efficacy of lenalidomide maintenance was not diminished by prior exposure to lenalidomide; in fact, the best outcomes were achieved when lenalidomide was given as both induction and maintenance. This is similar to results seen in previous lenalidomide maintenance studies, which showed significant heterogeneity of effect of lenalidomide maintenance with outcomes favoring those who had received lenalidomide induction. ${ }^{20,38}$ This suggests that patients with disease sensitive to immunomodulation with lenalidomide will continue to benefit from its continued use, perhaps as the maintenance therapy targets quiescent cells as they come out of cycle.

We noted that patients receiving CRD and observation appeared to have slightly inferior PFS than patients receiving CTD and observation. This was not due to any apparent difference in early discontinuation of therapy or dose 
Table 3. Adverse events according to induction regimen (safety population*).

\begin{tabular}{lcc}
\hline Grade $\geq 3 \mathrm{AE}, \mathrm{n}(\%)$ & $\begin{array}{c}\text { CRID } \\
(n=1,010)\end{array}$ & $\begin{array}{c}\text { CID } \\
(n=1,004)\end{array}$ \\
Neutropenia & $225(22.3)$ & $117(11.7)$ \\
Anemia & $97(9.6)$ & $67(6.7)$ \\
\hline Thrombocytopenia & $46(4.5)$ & $17(1.7)$ \\
Diarrhea & $26(2.6)$ & $10(1.0)$ \\
\hline Constipation & $8(0.8)$ & $19(1.9)$ \\
Peripheral sensory neuropathy & $6(0.6)$ & $15(1.5)$ \\
\hline Peripheral motor neuropathy & $5(0.5)$ & $14(1.4)$ \\
AEs of interest & CRiD & CIID \\
(ainy grade), n (\%) & $(n=1,010)$ & $(n=1,004)$ \\
Peripheral sensory neuropathy & $251(24.9)$ & $452(45.0)$ \\
Peripheral motor neuropathy & $87(8.6)$ & $163(16.2)$ \\
\hline Deep vein thrombosis & $58(5.7)$ & $48(4.8)$ \\
Pulmonary embolism & $32(3.2)$ & $49(4.9)$ \\
\hline Other thrombosis/embolism & $8(0.8)$ & $11(1.1)$ \\
\hline
\end{tabular}

*The safety population included all randomly assigned patients who received one or more doses of the induction or maintenance regimen. $\mathrm{AE}$ : adverse event; CRD: cyclophosphamide, lenalidomide, and dexamethasone; CTD: cyclophosphamide, thalidomide, and dexamethasone.

modifications and so is difficult to explain. The PFS difference is small, not statistically significant and may have occurred by chance. In the analysis of OS the reverse pattern was seen with patients receiving CRD and observation having an apparent improved OS compared to those receiving CTD and observation.

The results of Myeloma XI are likely to reflect the true impact of the CRD combination in clinical practice because of the limited exclusion criteria for the study population. Notably, there were no age restrictions for the intensive pathway, allowing older but fit patients to undergo ASCT. The median age in this group was 61 years, and patients up to the age of 75 years were included. In contrast, most previous studies of ASCT have excluded patients aged over 65 or 70 years. Evidence suggests that fit patients aged $>65$ years can benefit from ASCT, especially when combined with regimens containing novel agents. ${ }^{3,39,40}$ Our approach may also explain the relatively lower proportion of patients proceeding to ASCT in this study than in other studies of induction therapy which are usually limited only to patients under the age of 65 . The most common reason for patients not proceeding to stem-cell transplant was given as "patient not fit/clinician's decision" suggesting that clinicians may have initially entered patients in the transplant-eligible pathway of the study as a 'trial of fitness' so as not to limit their options prior to withdrawing the patient nearer the time of transplantation.

In addition, the proportion of patients with ISS stage III disease $(24 \%)$ in the present study was slightly higher than that in some recent studies of induction therapy., 10,31,35 Cytogenetic abnormalities, such as $t(4 ; 14), t(14 ; 16)$, and del(17p), are important prognostic markers, and should therefore be investigated in all patients with multiple myeloma according to the International Myeloma Working Group molecular classification. ${ }^{41}$ Although cytogenetic data were only available for $41 \%$ of patients in our study, this percentage is comparable to that in other trials of patients with newly diagnosed multiple myeloma. ${ }^{42}$
While three-drug induction regimens are generally more effective than two-drug regimens, they may also be more toxic. ${ }^{10,11}$ In the Myeloma XI trial, the safety results for CRD and CTD were consistent with the known safety profiles of these agents. Notably, rates of peripheral neuropathy were lower with CRD than with CTD. An important safety concern with lenalidomide treatment in patients with newly diagnosed multiple myeloma is the risk of SPM. ${ }^{43}$ In this population of transplant-eligible patients, the overall 3-year cumulative incidence of invasive SPM was low $(2.2 \%$; $95 \%$ CI: $1.5-3.0)$ and the type of induction therapy used did not appear to affect the SPM incidence rate. Safety results for lenalidomide maintenance compared to observation, including SPM incidence, have been previously published. ${ }^{23,24}$ Despite the risks associated with continued active therapy, registry data suggest that health-related quality of life of patients receiving lenalidomide maintenance is similar to that of patients receiving no maintenance..$^{45}$

In summary, induction therapy with CRD improved PFS and OS compared with CTD in transplant-eligible patients with newly diagnosed multiple myeloma. The best results were achieved when patients received both lenalidomide-based induction therapy and lenalidomide maintenance.

\section{Disclosures}

GHJ has received honoraria from and provided consultancy and speakers bureau services for Roche, Amgen, Janssen, and Merck Sharp and Dohme; and has received honoraria, travel support and research funding from and provided consultancy and speakers bureau services for Celgene Corporation and Takeda. $F E D$ reports consultancy for and honoraria from Amgen, AbbVie, Takeda, Janssen and Roche; and consultancy for and honoraria and research funding from Celgene Corporation. CP reports consultancy for and travel support from Amgen and Takeda Oncology; honoraria and travel support from Janssen; and consultancy for and honoraria and research funding from Celgene Corporation. DAC, AS, CC and $A W$ have received research funding from Celgene Corporation, Amgen, Merck Sharp and Dohme. JRJ has received honoraria and research funding from Celgene Corporation. BK reports consultancy and speakers bureau services for and travel support from Celgene Corporation, Takeda, and Janssen. MG has received travel support and research funding from and participated in speakers bureau for Janssen; has received travel support from Takeda; and travel support and research funding from Novartis. CDW has received honoraria and travel support from and participated in speakers bureau for Takeda, Janssen and Celgene Corporation; has received honoraria from Novartis; and has participated in speakers bureau for and received honoraria from Novartis. KK has received travel support and research funding from Celgene Corporation and Janssen. JL has provided consultancy services for Janssen; received travel support from Novartis; honoraria and travel support from Takeda; has acted as a consultant for and received travel support from Bristol-Myers Squibb; and reports consulting for and honoraria and travel support from Celgene Corporation. DA and NHR have nothing to disclose. SS reports travel support from Celgene Corporation and Janssen; speaker services for Pfizer; and meeting sponsorship from AbbVie. MWJ reports consultancy for and honoraria, travel support, and research funding from Janssen; consultancy for and honoraria and research funding from Celgene Corporation; consultancy for and honoraria from Novartis; and consultancy for and honoraria and travel support from Takeda and Amgen GC repports con- 
sultancy for and honoraria from Glycomimetics and BristolMyers Squibb; consultancy and speakers bureau services for and honoraria from Sanofi; and consultancy and speakers bureau services for and honoraria and research funding rom Takeda, Celgene Corporation, Janssen and Amgen. MFK reports consulting for Chugai; consultancy for and honoraria from Janssen and Amgen; consultancy for and travel support from Takeda and Bristol-Myers Squibb; and consultancy for and honoraria and research funding from Celgene Corporation. MTD has equity ownership or membership on the board of directors or advisory committees of Abingdon Health. RGO has received honoraria and travel support from Takeda; provided consultancy services for and received travel support from Janssen; and acted as a consultant for and received honoraria and research funding from Celgene Corporation. WMG reports consultancy services for and research funding from Celgene Corporation; research funding from Amgen, Merck Sharp and Dohme and honoraria from Janssen. GJM reports research funding from Janssen; consultancy for and honoraria from Bristol-Myers Squibb, Takeda, Roche, Amgen, GlaxoSmith Kline and Karyopharm; and consultancy for and honoraria and research funding from Celgene Corporation.

\section{Contributions}

GHJ, FED, NHR and GJM were the chief investigators; GHJ, FED, NHR, WMG and GJM designed the trial and developed the protocol; DAC, AS and WMG developed and carried out the statistical analysis; GHJ, FED, CP, JRJ, BK, MG, CDW, KK, $J L, D A, S S, M W J, G C, N H R, M F K, R G O$, and GJM participated in recruitment of patients; MFK, MTD, RGO, and GJM coordinated the central laboratory investigations; $C C$ and $A W$ coordinated the data collection and regulatory and governance requirements; GHJ, FED, CP, DAC, AS, MFK, MTD, RGO, WMG and GJM analyzed and interpreted the data; GHJ, FED, $C P, D A C, A S$ and GJM developed the first drafts of the manuscript. All authors contributed to the review and amendments of the manuscript for important intellectual content and approved the final version for submission.

\section{Acknowledgments}

We thank all the patients at centers throughout the UK whose willingness to participate made this study possible. We are grateful to the UK National Cancer Research Institute
Haematological Oncology Clinical Studies Group, UK Myeloma Research Alliance, and to all principal investigators, sub-investigators, and local center staff for their dedication and commitment to recruiting patients to the study. We thank the members of the Myeloma XI Trial Steering Committee and Data Monitoring and Ethics Committee. The support of the Clinical Trials Research Unit at the University of Leeds was essential to the successful running of the study; we thank all their staff who have contributed, past and present. Central laboratory analysis was performed at the Institute of Immunology and Immunotherapy, University of Birmingham; the Institute of Cancer Research, London; and the Haematological Malignancy Diagnostic Service, St James's University Hospital, Leeds. We are very grateful to the laboratory teams for their contribution to the study. We also acknowledge support from the National Institute of Health Biomedical Research Centre at the Royal Marsden Hospital and the Institute of Cancer Research. The authors received editorial support from Excerpta Medica, funded by the University of Leeds.

\section{Funding}

The primary financial support was from Cancer Research UK [C1298/A10410]. Unrestricted educational grants from Celgene Corporation, Amgen, and Merck Sharp and Dohme, and funding from Myeloma UK supported trial coordination and laboratory studies. The authors are solely responsible for study design, data collection, data analysis and interpretation, writing, and decisions about publication submission; no funder had any role in these aspects of the trial. Trial data were accessible to all authors. The corresponding author had full access to all of the data and the final responsibility to submit for publication.

\section{Data sharing statement}

De-identified participant data will be made available when all trial primary and secondary endpoints have been met. Any requests for trial data and supporting material (data dictionary, protocol, and statistical analysis plan) will be reviewed by the trial management group in the first instance. Only requests which have a methodologically sound proposal and whose proposed use of the data has been approved by the independent trial steering committee will be considered. Proposals should be directed to the corresponding author in the first instance; to gain access, data requestors will need to sign a data access agreement.

\section{References}

1. Kumar SK, Rajkumar SV, Dispenzieri A, et al. Improved survival in multiple myeloma and the impact of novel therapies. Blood. 2008;111(5):2516-2520.

2. Kristinsson SY, Anderson WF, Landgren $\mathrm{O}$. Improved long-term survival in multiple myeloma up to the age of 80 years. Leukemia. 2014;28(6):1346-1348.

3. Kumar SK, Dispenzieri A, Lacy $M Q$, et al. Continued improvement in survival in multiple myeloma: changes in early mortality and outcomes in older patients. Leukemia. 2014;28(5):1122-1128.

4. Child JA, Morgan GJ, Davies FE, et al. High-dose chemotherapy with hematopoietic stem-cell rescue for multiple myeloma. N Engl J Med. 2003;348(19):1875-1883.

5. Moreau P, Avet-Loiseau H, Facon T, et al. Bortezomib plus dexamethasone versus reduced-dose bortezomib, thalidomide plus dexamethasone as induction treatment before autologous stem cell transplantation in newly diagnosed multiple myeloma. Blood. 2011;118(22):5752-5758.

6. Palumbo A, Cavallo F, Gay F, et al. Autologous transplantation and maintenance therapy in multiple myeloma. $\mathrm{N}$ Engl J Med. 2014;371(10):895-905.

7. Gay F, Oliva S, Petrucci MT, et al. Chemotherapy plus lenalidomide versus autologous transplantation, followed by lenalidomide plus prednisone versus lenalidomide maintenance, in patients with multiple myeloma: a randomised, multicentre, phase 3 trial. Lancet Oncol. 2015; 16(16):1617-1629

8. Stadtmauer EA, Pasquini MC, Blackwell B, et al. Autologous transplantation, consolidation, and maintenance therapy in multiple myeloma: results of the BMT CTN 0702 trial. J Clin Oncol. 2019;37(7):589-597.

9. Attal M, Lauwers-Cances V, Hulin C, et al. Lenalidomide, bortezomib, and dexamethasone with transplantation for myeloma. N Engl J Med. 2017;376(14):1311-1320.

10. Cavo M, Tacchetti P, Patriarca F, et al.
Bortezomib with thalidomide plus dexamethasone compared with thalidomide plus dexamethasone as induction therapy before, and consolidation therapy after, double autologous stem-cell transplantation in newly diagnosed multiple myeloma: a randomised phase 3 study. Lancet. 2010;376(9758):2075-2085.

11. Davies FE, Wu P, Jenner M, et al. The combination of cyclophosphamide, velcade and dexamethasone induces high response rates with comparable toxicity to velcade alone and velcade plus dexamethasone. Haematologica. 2007;92(8):1149-1150.

12. Lopez-Girona A, Mendy D, Ito $\mathrm{T}$, et al. Cereblon is a direct protein target for immunomodulatory and antiproliferative activities of lenalidomide and pomalidomide. Leukemia. 2012;26(11):2326-2335.

13. Gandhi AK, Kang J, Havens CG, et al Immunomodulatory agents lenalidomide and pomalidomide co-stimulate $T$ cells by inducing degradation of $\mathrm{T}$ cell repressors Ikaros and Aiolos via modulation of the E3 
ubiquitin ligase complex CRL4. Br J Haematol. 2014;164(6):811-821.

14. Kronke J, Udeshi ND, Narla A, et al. Lenalidomide causes selective degradation of IKZF1 and IKZF3 in multiple myeloma cells. Science. 2014;343(6168):301-305

15. Bjorklund CC, Lu L, Kang J, et al. Rate of CRL4(CRBN) substrate Ikaros and Aiolos degradation underlies differential activity of lenalidomide and pomalidomide in multiple myeloma cells by regulation of c-Myc and IRF4. Blood Cancer J. 2015;5:e354.

16. Wu P, Davies FE, Horton C, et al. The combination of cyclophosphomide, thalidomide and dexamethasone is an effective alternative to cyclophosphamide - vincristine - doxorubicin - methylprednisolone as induction chemotherapy prior to autologous transplantation for multiple myeloma: a case-matched analysis. Leuk Lymphoma. 2006;47(11):2335-2338.

17. Morgan GJ, Davies FE, Gregory WM, et al. Cyclophosphamide, thalidomide, and dexamethasone as induction therapy for newly diagnosed multiple myeloma patients destined for autologous stem-cell transplantation: MRC Myeloma IX randomized trial results. Haematologica. 2012;97(3):442450.

18. Morgan GJ, Davies FE, Gregory WM, et al. Long-term follow-up of MRC Myeloma IX Trial: survival outcomes with bisphosphonate and thalidomide treatment. Clin Cancer Res. 2013;19(21):6030-6038.

19. Attal M, Lauwers-Cances V, Marit G, et al. Lenalidomide maintenance after stem-cell transplantation for multiple myeloma. N Engl J Med. 2012;366(19):1782-1791.

20. McCarthy PL, Owzar K, Hofmeister CC, et al. Lenalidomide after stem-cell transplantation for multiple myeloma. N Engl J Med. 2012;366(19):1770-1781.

21. Morgan GJ, Gregory WM, Davies FE, et al. The role of maintenance thalidomide therapy in multiple myeloma: MRC Myeloma IX results and meta-analysis. Blood. 2012; 119(1):7-15.

22. Jackson GH, Davies FE, Pawlyn C, et al. Response-adapted intensification with cyclophosphamide, bortezomib, and dexamethasone versus no intensification in patients with newly diagnosed multiple myeloma (Myeloma XI): a multicentre, open-label, randomised, phase 3 trial. Lancet Haematol. 2019;6(12):e616-e629.

23. Jackson GH, Davies FE, Pawlyn C, et al. Lenalidomide maintenance versus observation for patients with newly diagnosed multiple myeloma (Myeloma XI): a multicentre, open-label, randomised, phase 3 trial. Lancet Oncol. 2019;20(1):57-73

24. Morgan GJ, Davies FE, Gregory WM, et al. Long-term follow-up of MRC Myeloma IX trial: survival outcomes with bisphosphonate and thalidomide treatment. Clin Cancer Res. 2013;19(21):6030-6038.
25. Kumar SK, Lacy MQ, Hayman SR, et al. Lenalidomide, cyclophosphamide and dexamethasone (CRd) for newly diagnosed multiple myeloma: results from a phase 2 trial. Am J Hematol. 2011;86(8):640-645.

26. Morgan GJ, Schey SA, Wu P, et al. Lenalidomide (Revlimid), in combination with cyclophosphamide and dexamethasone (RCD), is an effective and tolerated regimen for myeloma patients. $\mathrm{Br}$ Haematol. 2007;137(3):268-269.

27. Gay F, Hayman SR, Lacy MQ, et al. Lenalidomide plus dexamethasone versus thalidomide plus dexamethasone in newly diagnosed multiple myeloma: a comparative analysis of 411 patients. Blood. 2010;115(7):1343-1350.

28. Luo J, Gagne JJ, Landon J, et al Comparative effectiveness and safety of thalidomide and lenalidomide in patients with multiple myeloma in the United States of America: a population-based cohort study. Eur J Cancer. 2017;70:22-33.

29. Zweegman S, van der Holt B, Mellqvist $\mathrm{UH}$, et al. Melphalan, prednisone, and lenalidomide versus melphalan, prednisone, and thalidomide in untreated multiple myeloma. Blood. 2016;127(9):1109 1116.

30. Stewart AK, Jacobus S, Fonseca R, et al. Melphalan, prednisone, and thalidomide vs melphalan, prednisone, and lenalidomide (ECOG E1A06) in untreated multiple myeloma. Blood. 2015;126(11):1294-1301.

31. Sonneveld P, Schmidt-Wolf IG, van de Holt $\mathrm{B}$, et al. Bortezomib induction and maintenance treatment in patients with newly diagnosed multiple myeloma: results of the randomized phase III HOVON-65/ GMMG-HD4 trial. J Clin Oncol. 2012;30(24):2946-2955.

32. Mai EK, Bertsch U, Dürig J, et al. Phase III trial of bortezomib, cyclophosphamide and dexamethasone (VCD) versus bortezomib, doxorubicin and dexamethasone (PAd) in newly diagnosed myeloma. Leukemia. 2015;29(8):1721-9.

33. Rosinol L, Oriol A, Teruel AI, et al. Superiority of bortezomib, thalidomide, and dexamethasone (VTD) as induction pretransplantation therapy in multiple myeloma: a randomized phase 3 PETHEMA/GEM study. Blood. 2012;120(8):15891596

34. Cavo M, Pantani L, Pezzi A, et al Bortezomib-thalidomide-dexamethasone (VTD) is superior to bortezomibcyclophosphamide-dexamethasone (VCD) as induction therapy prior to autologous stem cell transplantation in multiple myeloma. Leukemia. 2015;29(12):2429-2431.

35. Kumar S, Flinn I, Richardson PG, et al. Randomized, multicenter, phase 2 study (EVOLUTION) of combinations of bortezomib, dexamethasone, cyclophosphamide, and lenalidomide in previously untreated multiple myeloma. Blood. 2012; 119(19):4375-4382.

36. Moreau P, Attal M, Hulin C, et al Bortezomib, thalidomide, and dexamethasone with or without daratumumab before and after autologous stem-cell transplantation for newly diagnosed multiple myeloma (CASSIOPEIA): a randomised, openlabel, phase 3 study. Lancet. 2019; 394(10192):29-38.

37. McCarthy PL, Holstein SA, Petrucci MT, et al. Lenalidomide maintenance after autologous stem-cell transplantation in newly diagnosed multiple myeloma: a metaanalysis. J Clin Oncol. 2017;35(29):32793289

38. Holstein SA, Jung SH, Richardson PG, et al Updated analysis of CALGB (Alliance) 100104 assessing lenalidomide versus placebo maintenance after single autologous stem-cell transplantation for multiple myeloma: a randomised, double-blind, phase 3 trial. Lancet Haematol. 2017; 4(9): e431-e442.

39. Ozaki S, Harada T, Saitoh T, et al. Survival of multiple myeloma patients aged 65-70 years in the era of novel agents and autologous stem cell transplantation. A multicenter retrospective collaborative study of the Japanese Society of Myeloma and the European Myeloma Network [Multicenter Study]. Acta Haematol. 2014;132(2):211 219.

40. Minoia C, Pisapia G, Palazzo G, et al Impact of novel agents followed by autologous hematopoietic stem cell transplantation for multiple myeloma patients aged 65 years or older: a retrospective single Institutional analysis. Bone Marrow Transplant. 2015;50(11):1486

41. Fonseca R, Bergsagel PL, Drach J, et al. International Myeloma Working Group molecular classification of multiple myeloma: spotlight review. Leukemia. 2009; 23(12):2210-2221

42. Avet-Loiseau H, Facon T. Front-line therapies for elderly patients with transplantineligible multiple myeloma and high-risk cytogenetics in the era of novel agents. Leukemia. 2018;32(6):1267-1276.

43. Palumbo A, Bringhen S, Kumar SK, et al Second primary malignancies with lenalidomide therapy for newly diagnosed myeloma: a meta-analysis of individual patient data. Lancet Oncol. 2014;15(3):333-342.

44. Jones JR, Cairns DA, Gregory WM, et al. Second malignancies in the context of lenalidomide treatment: an analysis of 2732 myeloma patients enrolled to the Myeloma XI trial. Blood Cancer J. 2016;6(12):e506.

45. Abonour R, Wagner L, Durie BGM, et al Impact of post-transplantation maintenance therapy on health-related quality of life in patients with multiple myeloma: data from the Connect $($ MM Registry. Ann Hematol. 2018;97(12):2425-2436. 